\title{
The dose-response effect of fruit and vegetable intake on ambulatory blood pressure, in healthy individuals at high risk of cardiovascular disease: a randomised controlled trial
}

\author{
C. T. McEvoy ${ }^{1}$, I. R. Wallace ${ }^{2}$, L. L. Hamill ${ }^{1}$, C. E. Neville ${ }^{1}$, S. J. Hunter ${ }^{2}$, J. V. Woodside $^{1}$, \\ I. S. Young and M. C. McKinley ${ }^{1}$ \\ ${ }^{1}$ Nutrition and Metabolism Group, Centre for Public Health, Queen's University Belfast, BT12 6BJ and \\ ${ }^{2}$ Regional Centre for Endocrinology \& Diabetes, Royal Victoria Hospital, Belfast, BT12 6BA, UK
}

Ambulatory blood pressure monitoring (ABPM) has greater statistical power to detect true differences in blood pressure values in clinical trials and has greater prognostic significance in cardiovascular target organ damage when compared to clinic measured blood pressure ${ }^{(1)}$. The aim of this study was to examine the dose-response effect of fruit and vegetable (FV) intake on 24-hour AMBP in healthy, overweight adults at high cardiovascular disease risk. Ethical approval was received from the Office for Research Ethics Committees Northern Ireland.

Following a 4 week run-in period, during which FV was limited to $<2$ portions per day, participants were randomized to consume either 2, 4 or 7 FV portions daily for the next 12 weeks. AMBP was measured over 24-hours using a validated Meditech ABPM-04 system (P.M.S. Ltd, UK) at baseline and post-intervention. Dietary compliance was monitored using completed 4-day food diaries and by measuring a panel of venous blood dietary biomarkers.

In total, 87 participants (64\% male; $36 \%$ female) with a mean age 56 years (SD 6.0 years) completed the study. Reported FV intake in the 2, 4 and 7 portion groups was 1.8, 3.8 and 7.0 portions per day $(p<0.001)$ respectively. Serum lutein also increased linearly across the groups $(P$ for Trend $<0.001)$. Body weight remained stable throughout the intervention period $(p=0.77)$. Mean systolic/diastolic blood pressure was $127.8 / 76.6 \mathrm{mmHg}$ (SD 12.1/8.8) with no significant difference between the groups at baseline. Statistical analysis was performed using one-way analysis of variance with fitted linear trend.

\begin{tabular}{|c|c|c|c|c|c|c|c|c|c|c|}
\hline & \multicolumn{3}{|c|}{2 portions/d $(n=28 \max )$} & \multicolumn{3}{|c|}{$\mathbf{4}$ portions/d $(n=29 \max )$} & \multicolumn{3}{|c|}{7 portions $/ \mathbf{d}(n=30 \max )$} & \multirow[b]{2}{*}{$\mathbf{P}_{\text {Trend }}$} \\
\hline & $\begin{array}{l}\text { *Mean } \\
\text { change }\end{array}$ & $\begin{array}{c}\text { Mean change } \\
95 \% \text { CI }\end{array}$ & $\begin{array}{c}\text { Within group } \\
p \text { value }\end{array}$ & $\begin{array}{l}* \text { Mean } \\
\text { change }\end{array}$ & $\begin{array}{c}\text { Mean change } \\
95 \% \mathrm{CI}\end{array}$ & $\begin{array}{c}\text { Within group } \\
p \text { value }\end{array}$ & $\begin{array}{l}\text { *Mean } \\
\text { change }\end{array}$ & $\begin{array}{l}\text { Mean change } \\
95 \% \mathrm{CI}\end{array}$ & $\begin{array}{c}\text { Within group } \\
p \text { value }\end{array}$ & \\
\hline $\begin{array}{l}\text { ABPM Systolic }(\mathrm{mmHg}) \\
\text { ABPM Diastolic }(\mathrm{mmHg})\end{array}$ & $\begin{array}{l}-3.0 \\
-2.1\end{array}$ & $\begin{array}{l}-7.66,1.66 \\
-5.54,1.40\end{array}$ & $\begin{array}{l}0.20 \\
0.23\end{array}$ & $\begin{array}{l}+1.2 \\
+0.5\end{array}$ & $\begin{array}{l}-2.58,4.93 \\
-2.31,3.21\end{array}$ & $\begin{array}{l}0.53 \\
0.74\end{array}$ & $\begin{array}{l}-2.0 \\
-1.28\end{array}$ & $\begin{array}{l}-5.25,1.25 \\
-3.90,1.35\end{array}$ & $\begin{array}{l}0.22 \\
0.33\end{array}$ & $\begin{array}{l}0.87 \\
0.82\end{array}$ \\
\hline
\end{tabular}

*Mean change $=$ mean post-intervention - mean baseline value.

Despite good compliance with the study protocol, we found no evidence of a dose-response effect of FV intake on gold standard ABPM. Our results agree with findings from recent FV intervention trials ${ }^{(2,3)}$. In contrast, the Dietary Approaches to Stop Hypertension trial provides convincing evidence for implementation of a broader dietary approach in relation to blood pressure reduction ${ }^{(4)}$.

1. Palatini P (2000) Curr Hypertens Rep 2, 362-369.

2. Berry SE, Mulla UZ, Chowienczyk PJ et al. (2010) Br J Nutr 104, 1839-1847.

3. Dohadwala MM, Hamburg NM, Holbrook et al. (2010) Am J Clin Nutr 92, 1052-1059.

4. Appel LJ, Champagne CM, Harsha DW et al. (1997) N Engl J Med 336, 1117-1124. 